\title{
Velocity variability of semiregular and irregular variables
}

\author{
T. Lebzelter ${ }^{1}$ and K. H. Hinkle ${ }^{2}$ \\ 1 Institut für Astronomie, Universität Wien, Türkenschanzstr. 17, 1180 Vienna, Austria \\ 2 National Optical Astronomy Observatory ${ }^{\star}, 950$ N. Cherry Avenue, PO Box 26732, Tucson, Arizona 85726, USA
}

Received 17 April 2002 / Accepted 11 July 2002

\begin{abstract}
We compare velocities from near infrared lines with center-of-mass velocities for a sample of semiregular and irregular variables to search for indications for a convective blueshift. It is shown that a general blueshift is present but that the light variability is obviously not dominated by convective cells but rather by stellar pulsation. All stars of our sample show a similar shape and amplitude in the velocity variations. Long secondary periods are a common feature in these objects and strongly influence the measured velocity shifts. The star V366 Aql is found to be the first SRV showing line doubling.
\end{abstract}

Key words. stars: variables: general - stars: late-type - stars: AGB and post-AGB - stars: atmospheres

\section{Introduction}

A basic understanding of pulsating stars stems from detailed measurements of brightness and radial velocity as a function of pulsation phase with a knowledge of distance required to calibrate these numbers. For the large amplitude AGB variable stars measurements of these quantities have proven difficult (see e.g. van Belle et al. 1996 and Hinkle 1978). However, most AGB variables have small amplitudes, with visual amplitudes of only a few tenths of a magnitude to a few magnitudes. The periods of these "semi-regular variables" (SRVs), are typically in the range 30 to 200 days rather than a year or more for the large amplitude mira variables. The variation of the SRVs is typically less periodic than the miras, therefore these objects have been classified semiregular (SR) or irregular (Lb) variables. Semiregular variables are classically separated into two groups a and b. SRa variables show a rather well expressed periodicity and differ from the miras mainly in their smaller light amplitude. SRb stars show a less expressed periodicity. The SRVs have been recognized for a long time but good measurements of even the brightness variations are only recently available. As a result the mechanism causing their light (and velocity) variability remains poorly understood. Identification of this mechanism is important for defining the blue edge of the AGB instability region in the Hertzsprung-Russell diagram (HRD) (e.g. Gautschy 1999).

Send offprint requests to: T. Lebzelter, e-mail: lebzelter@astro.univie.ac.at

* Operated by the Association of Universities for Research in Astronomy, Inc. under cooperative agreement with the National Science Foundation.
Weak to moderate strength lines in the 1-2 $\mu \mathrm{m}$ infrared spectrum of AGBs have contribution curves (Edmonds 1969; Loidl et al. 1999) indicating formation in the "photosphere". Lines formed in the visual (e.g. Hinkle \& Barnes 1979) and in the $4 \mu \mathrm{m}$ range (Lebzelter et al. 2001) are formed in more extended atmospheric layers. Fortunately the near infrared is rich with weak to moderate strength lines, especially the CO $\Delta v=3$ vibration-rotational lines located around $1.6 \mu \mathrm{m}$. These lines have been used to probe stellar pulsation in miras (e.g. Hinkle et al. 1982). The same lines have also been used to analyze SR and Lb variables by Hinkle et al. (1997) and Lebzelter (1999).

The result of these investigations was that all miras exhibit similar velocity variations clearly correlated with the visual light cycle. The velocity curve is s-shaped and discontinuous with line doubling around maximum phase (Lebzelter \& Hinkle 2002). This is an indicator of a shock front running through the atmosphere. All miras show a similar velocity amplitude ranging between 20 and $30 \mathrm{~km} \mathrm{~s}^{-1}$. The semiregular and irregular variables investigated so far all show velocity amplitudes of a few $\mathrm{km} \mathrm{s}^{-1}$ except for three stars (W Hya, X Oph, $\mathrm{XMon}$ ) which show velocity amplitudes of 10 to $15 \mathrm{kms}^{-1}$. However, the classification of these stars as SRVs is not clear.

To derive pulsational properties it is critical to set the measured velocities into relation with the systemic velocity. The most reliable source of center-of-mass (COM) velocities of AGB stars is radio measurement of circumstellar microwave lines of CO. However, for only a small number of the SRVs and Lbs investigated in the near infrared have microwave lines been detected. Comparing the infrared velocities of SR and Lb stars with known COM velocity Lebzelter (1999) found that the IR velocities are blue shifted for most or even all of the light cycle. Lebzelter suggested that this phenomenon might be the 
result of a convective blue shift similar to the blue shift caused by granules on the solar surface (Dravins 1987) or on F-G-K main sequence stars (Allende Prieto et al. 2002) but no final conclusion could be drawn from the existing data material. On the other hand, Wallerstein \& Dominy (1988) found no systematic velocity shift in a sample of four SRVs. This difference may be explained as Wallerstein \& Dominy used low excitation 2-0 CO lines instead of the second overtone lines. It has been shown that for miras (e.g. Hinkle et al. 1982) these lines are almost always found near the COM velocity. This was also found by Lebzelter (1999) for the SRV g Her. A significant problem is that the small number of objects does not allow an understanding of the blue shift detected in the sample of Lebzelter (1999). Recently, Hinkle et al. (2002) have shown that the long period variations found in the light curves of a large fraction of SRVs are in many cases also present in the velocity variations. These variations have to be taken into account when analyzing this question.

The purpose of this paper is twofold: one goal is to study in more detail the observed blue shift in the velocity curves of semiregular variables. Due to the long periods of the stars velocity time series of individual objects was not a feasible way to attack the problem. Instead we measured the velocity of near infrared (NIR) CO lines for a large sample of stars with known center-of-mass velocities at two epochs. The second goal was to better characterize the shape and amplitude of the velocity variations. Both aspects are critical for understanding the variability mechanism in small amplitude variables.

\section{Observations and data reduction}

\subsection{Definition of the sample}

Our sample consists of two groups: a core sample was defined including variables of type $\mathrm{SRa}, \mathrm{SRb}$ and $\mathrm{Lb}$, for which a stellar velocity has been derived from circumstellar $\mathrm{CO}$ radio observations. We used the compilation published by Kerschbaum \& Olofsson (1999) to select these stars. Kerschbaum \& Olofsson estimate that the velocity values from the radio $\mathrm{CO}$ lines are uncertain by about 1 to $2 \mathrm{~km} \mathrm{~s}^{-1}$, highly depending on the $S / N$ ratio. Recent observations of three further SRVs (Olofsson et al. 2002) have been added to this list. The core sample consisted of 36 stars. 25 of them were observed at two epochs (see below).

The sample was extended by 34 further semiregular and irregular variables selected to cover a large range in period and amplitude. These stars were observed at two epochs to derive a lower limit for the amplitude of the velocity variation. A few further stars could be observed only once and will not be discussed in this paper. The whole sample is listed in Tables 1 and 2.

\subsection{Velocity data}

High resolution $(\lambda / \Delta \lambda \sim 50000)$ near infrared spectra of the program stars were obtained with the Phoenix spectrograph (Hinkle et al. 1998) mounted at the $f / 15$ Cassegrain focus of the Kitt Peak National Observatory $2.1 \mathrm{~m}$ telescope. There were two observing runs (April 2000, July 2000) with seven usable nights in total. The observations consisted of $\sim 80 \AA$ of spectrum centered at $15620 \AA$. This wavelength region contains a selection of CO 5-2 and 6-3 s overtone lines and a number of Fe lines (Hinkle et al. 1995). As mentioned above, these lines already have been used successfully for several previous velocity monitoring programs of AGB stars. For almost all stars we achieved a $S / N$ ratio of 50 or better. The wavelength range selected is free of strong atmospheric lines. Wavelength calibration was done using primary velocity standard stars.

Stellar velocities have been measured by cross correlation of the sample stars with velocity standards using a slightly modified version of the program described in Lebzelter (1999). Two to three velocity standards and a number of $\mathrm{G}$ and $\mathrm{K}$ giants as secondary standards have been observed several times during each night. Each primary standard was typically observed 4 to 5 times per night. Secondary standards were measured 1 to 4 times during the night. The standard stars are listed in Table 3. The primary standards are IAU radial velocity standards taken from a list of Skuljan et al. (2000). The derived velocities of the secondary standards are in good agreement with measurements found in the literature (Duflot et al. 1995; de Medeiros \& Mayor 1999). No velocity difference between the April and the July run was found for the $\mathrm{G}$ and $\mathrm{K}$ giants.

The velocity accuracy achieved was evaluated from the secondary velocity standards. From the spectral resolution and the use of the correlation technique one would expect a velocity resolution of about 100 to $200 \mathrm{~m} / \mathrm{s}$. However, as the Phoenix spectrograph was directly attached to the telescope, small shifts in the central wavelength during the night are inevitable. After careful data reduction the velocities of the secondary standards turned out to scatter by about $0.4 \mathrm{~km} \mathrm{~s}^{-1}$ within one run. The same scatter was also found when averaging over all nights. We therefore think that this value represents quite well the velocity uncertainty.

If more than one observation of a sample star was obtained during one of the runs, the velocities have been averaged. The scatter was in most cases similar to the scatter found for the secondary standards. Two stars showed a significantly larger scatter: CX Her $\left(1.5 \mathrm{~km} \mathrm{~s}^{-1}\right)$ and $\mathrm{g} \operatorname{Her}\left(1.5 \mathrm{~km} \mathrm{~s}^{-1}\right)$. As there is no indication of an error in the measurement of these stars, we assume that they varied within the three nights of an observing run. The resulting velocities at each epoch can be found in Tables 1 and 2.

The star V366 Aql shows a spectrum different from the other stars in the sample. This object is discussed in detail in the appendix.

\subsection{Photometric data}

In the case of semiregular variables the mean period given in the General Catalogue of Variable stars (GCVS, Kholopov et al. 1985-88) is not necessarily representative for the variability at a given time (Lebzelter et al. 1995; Kiss et al. 1999; Kerschbaum et al. 2001). To derive an approximate phase for the star, light curve monitoring parallel to the velocity observations is required. For 55 stars of our sample this monitoring was done with the Vienna Automatic Photometric Telescopes 
Table 1. Program stars - core sample. Star name (Cols. 1 and 2), variability type (Col. 3), period (Col. 4), velocity from Kerschbaum \& Olofsson 1999 (Col. 5) and the velocity from the two observing runs (Cols. 6 to 9) are listed. All velocities are heliocentric. Standard deviations are given in cases where more than one spectrum was obtained during an observing run. The number of observations is given in brackets. The period given is the period used in the analysis, i.e. modified from the literature value, if our own photometric data suggested a different period. Column 10 gives the V light amplitude from our APT data. Stars with a suspected second long period are marked in the last column (only for stars with APT data).

\begin{tabular}{|c|c|c|c|c|c|c|c|c|c|c|}
\hline \multirow{2}{*}{$\begin{array}{l}\mathrm{HD} / \mathrm{HIP} / \mathrm{SAO} \\
\text { number }\end{array}$} & \multirow[t]{2}{*}{ name } & \multirow{2}{*}{$\begin{array}{r}\text { variable } \\
\text { type }\end{array}$} & \multirow{2}{*}{$\begin{array}{r}\text { period } \\
\text { [d] }\end{array}$} & \multicolumn{5}{|c|}{ velocity $\left[\mathrm{km} \mathrm{s}^{-1}\right]$} & \multirow{2}{*}{$\begin{array}{c}V \text { ampl. } \\
\text { [mag] }\end{array}$} & \multirow{2}{*}{$\begin{array}{c}\text { long } \\
\text { period }\end{array}$} \\
\hline & & & & radio & April & $\sigma$ & July & $\sigma$ & & \\
\hline HD 2326 & UY Cet & $\mathrm{SRb}$ & 440 & 7.5 & - & & 7.6 & & 0.7 & \\
\hline HD 17446 & T Ari & SRa & 317 & 6.4 & - & & 7.9 & & & \\
\hline HD 40913 & V352 Ori & $\mathrm{Lb}$ & 115 & 38.8 & 40.2 & & - & & 0.3 & \\
\hline HD 70938 & FK Hya & $\mathrm{Lb}$ & 100 & 55.5 & 57.1 & $0.0(2)$ & - & & 0.6 & + \\
\hline HD 73844 & AK Hya & $\mathrm{SRb}$ & 75 & 35.4 & 38.3 & $0.4(2)$ & - & & 0.4 & \\
\hline HD 78420 & $\mathrm{CW} \mathrm{Cnc}$ & $\mathrm{Lb}$ & 100 & 25.1 & 24.1 & $0.4(3)$ & - & & 0.5 & \\
\hline HD 95384 & $\mathrm{R} \mathrm{Crt}$ & $\mathrm{SRb}$ & 160 & 18.9 & 18.4 & $0.4(3)$ & - & & 0.3 & \\
\hline HD 105266 & RW Vir & $\mathrm{Lb}$ & 116 & 22.0 & 23.6 & $0.8(2)$ & 23.7 & & 0.2 & + \\
\hline HD 108849 & BK Vir & $\mathrm{SRb}$ & 150 & 14.3 & 13.2 & $0.6(3)$ & 13.3 & & 0.9 & \\
\hline HD 110259 & Y UMa & $\mathrm{SRb}$ & 168 & 8.8 & 8.6 & $0.5(4)$ & 7.7 & & 0.6 & \\
\hline HD 113285 & RT Vir & $\mathrm{SRb}$ & 155 & 12.1 & 11.5 & $0.4(4)$ & 10.4 & $0.5(2)$ & 0.7 & + \\
\hline HD 114961 & SW Vir & $\mathrm{SRb}$ & 150 & -16.0 & -12.7 & $0.1(4)$ & -17.0 & $0.2(2)$ & 1.0 & \\
\hline HD 126327 & RX Boo & $\mathrm{SRb}$ & 157 & -11.7 & -13.3 & $0.7(3)$ & -13.8 & & 0.2 & \\
\hline HD 129004 & RV Boo & $\mathrm{SRb}$ & 137 & -6.5 & -4.5 & 0.7 (3) & -7.1 & & 0.9 & \\
\hline HD 139216 & $\tau^{4} \operatorname{Ser}$ & $\mathrm{SRb}$ & 100 & -29.2 & -22.8 & $0.8(3)$ & -23.3 & & 0.4 & \\
\hline HD 144205 & X Her & $\mathrm{SRb}$ & 95 & -89.9 & -91.7 & $0.3(3)$ & -93.1 & & 0.6 & \\
\hline HD 148783 & g Her & $\mathrm{SRb}$ & 89 & 1.3 & -0.6 & $1.5(3)$ & -0.8 & $0.2(2)$ & 0.4 & + \\
\hline HD 151187 & S Dra & $\mathrm{SRb}$ & 136 & -2.1 & -5.6 & $0.3(3)$ & -3.3 & & 0.4 & \\
\hline HD 152152 & AH Dra & $\mathrm{SRb}$ & 158 & 56.9 & 55.4 & $0.3(2)$ & 57.9 & & 0.6 & \\
\hline HD 160540 & TY Dra & $\mathrm{Lb}$ & 89 & -34.5 & -35.2 & $0.4(2)$ & -36.0 & & 0.4 & \\
\hline HD 168829 & TU Lyr & $\mathrm{Lb}$ & - & -10.1 & -12.5 & $0.1(2)$ & -10.9 & & 0.5 & \\
\hline HD 172885 & SY Lyr & $\mathrm{SRb}$ & 100 & 39.1 & 39.5 & $0.1(2)$ & 39.8 & & 0.4 & \\
\hline HD 175188 & UX Sgr & $\mathrm{SRb}$ & 100 & 5.5 & 4.7 & & 3.8 & & 0.4 & + \\
\hline HD 180004 & SZ Dra & $\mathrm{Lb}$ & 114 & -42.0 & -43.3 & & -44.0 & & 0.4 & + \\
\hline HD 184008 & AF Cyg & $\mathrm{SRb}$ & 93 & -15.2 & -17.0 & $0.3(2)$ & -17.6 & & 1.0 & \\
\hline HD 197892 & U Del & $\mathrm{SRb}$ & 205 & -22.2 & -21.2 & $0.5(2)$ & -21.3 & $0.4(2)$ & 0.3 & + \\
\hline HD 205730 & W Cyg & $\mathrm{SRb}$ & 131 & -14.3 & -14.5 & & -17.1 & & & \\
\hline HD 207076 & EP Aqr & $\mathrm{SRb}$ & 55 & -43.1 & -41.5 & & -42.4 & & 0.6 & \\
\hline HD 209598 & TW Peg & $\mathrm{SRb}$ & 929 & -23.4 & -22.6 & & -22.8 & $0.2(2)$ & 0.4 & + \\
\hline HD 209872 & SV Peg & $\mathrm{SRb}$ & 145 & -6.8 & -4.2 & & -7.3 & & & \\
\hline HD 224126 & RS And & SRa & 200 & -2.1 & - & & -2.1 & & 0.4 & \\
\hline HIP 43063 & EY Hya & $\mathrm{SRa}$ & 183 & 35.5 & 34.8 & $0.2(2)$ & - & & 0.7 & \\
\hline HIP 57504 & AZ UMa & $\mathrm{Lb}$ & - & -49.5 & -53.5 & $0.5(3)$ & -51.2 & & 0.8 & \\
\hline HIP 80259 & RY CrB & $\mathrm{SRb}$ & 90 & 20.4 & 19.5 & $0.4(2)$ & 18.2 & & 0.5 & + \\
\hline SAO 38115 & UX And & $\mathrm{SRb}$ & 400 & -18.7 & - & & -20.9 & & & \\
\hline SAO 116668 & FZ Hya & $\mathrm{Lb}$ & 134 & 0.1 & -3.1 & $0.0(2)$ & - & & 0.6 & \\
\hline- & CZ Ser & $\mathrm{Lb}$ & - & -31.5 & -29.9 & & - & & & \\
\hline
\end{tabular}

(APT, Strassmeier et al. 1997). Observations were taken in Johnson V. The monitoring covered a time span of 200 to 500 days. Typically, one data point was obtained every second night. The uncertainty of the measurements is of the order of $0.02 \mathrm{mag}$. Due to weather problems and limited access to the APT for this project, there are some gaps in the light curves. About half the sample was also part of a long time monitoring program at the APT so that much longer data sets exist for these stars (see e.g. Kerschbaum et al. 2001 for examples of the light curves obtained).

The light curves were analyzed using Period98 (Sperl 1998). If the derived "current" period differed significantly from the GCVS period, the APT period was used in the following data analysis instead. Where possible a period (i.e. a typical time scale of the variability) was also derived for the irregular variables. This allowed the inclusion of 9 irregular variables in the further analysis. In most cases the values we found for the SRVs were in good agreement with the GCVS values but for six stars a period strongly differing from the literature value has been found. Some of these cases are supported by other investigations (e.g. Kiss et al. 1999; Lebzelter \& Kiss 2001). Typical amplitudes of the light change of the short period variation have been derived and are listed in Tables 1 and 2. The APT data also clearly showed the existence of long secondary 
Table 2. Program stars - extended sample. Star name (Cols. 1 and 2), variability type (Col. 3), period (Col. 4) and the velocity from the two observing runs (Cols. 5 and 8 ) are listed. The period given is the period used in the analysis, i.e. modified from the literature value, if our own photometric data suggested a different period. All velocities are heliocentric.

\begin{tabular}{|c|c|c|c|c|c|c|c|c|c|}
\hline \multirow{2}{*}{$\begin{array}{l}\mathrm{HD} / \mathrm{HIP} / \mathrm{SAO} \\
\text { number }\end{array}$} & \multirow[t]{2}{*}{ name } & \multirow{2}{*}{$\begin{array}{r}\text { variable } \\
\text { type }\end{array}$} & \multirow{2}{*}{$\begin{array}{r}\text { period } \\
{[\mathrm{d}]}\end{array}$} & \multicolumn{4}{|c|}{ velocity $\left[\mathrm{km} \mathrm{s}^{-1}\right]$} & \multirow{2}{*}{$\begin{array}{c}V \text { ampl. } \\
{[\mathrm{mag}]}\end{array}$} & \multirow{2}{*}{$\begin{array}{l}\text { long } \\
\text { period }\end{array}$} \\
\hline & & & & April & $\sigma$ & July & $\sigma$ & & \\
\hline HD 99635 & AF Leo & $\mathrm{SRb}$ & 119 & 4.2 & $0.5(3)$ & 5.6 & & 0.3 & \\
\hline HD 101487 & AK Leo & $\mathrm{SRb}$ & 47 & -28.4 & $0.6(3)$ & -25.0 & & 0.2 & \\
\hline HD 102159 & TV UMa & $\mathrm{SRb}$ & 51 & 53.2 & $0.6(3)$ & 56.7 & & 0.3 & \\
\hline HD 107937 & FK Vir & $\mathrm{SRb}$ & 40 & 17.1 & $0.4(3)$ & 18.7 & & 0.2 & \\
\hline HD 108345 & SS Dra & $\mathrm{SRb}$ & 52 & 26.8 & $0.9(2)$ & 27.1 & & 0.3 & \\
\hline HD 115898 & V CVn & SRa & 192 & -9.0 & $0.2(4)$ & -5.9 & & 0.4 & \\
\hline HD 120806 & AY Vir & $\mathrm{SRb}$ & 193 & -49.0 & $0.4(2)$ & -49.8 & & 1.0 & \\
\hline HD 128333 & CH Boo & $\mathrm{Lb}$ & - & -24.5 & & -25.2 & & & \\
\hline HD 129355 & RW Boo & $\mathrm{SRb}$ & 58 & -10.2 & & -10.1 & & 0.3 & \\
\hline HD 130082 & UV Dra & SRa & - & -41.1 & & -45.3 & & & \\
\hline HD 132112 & FY Lib & $\mathrm{SRb}$ & 103 & 8.5 & $0.2(2)$ & 7.4 & & 0.4 & \\
\hline HD 134943 & FL Ser & $\mathrm{Lb}$ & - & -38.0 & & -36.7 & & & \\
\hline HD 135205 & Y Ser & SRa & 433 & -69.6 & & -71.3 & $0.1(3)$ & & \\
\hline HD 140155 & SW CrB & $\mathrm{SRb}$ & 100 & -20.0 & & -20.6 & & & \\
\hline HD 140297 & RR CrB & $\mathrm{SRb}$ & 55 & -59.6 & $0.5(2)$ & -59.2 & & 0.3 & \\
\hline HD 142143 & ST Her & $\mathrm{SRb}$ & 148 & -25.3 & $0.2(2)$ & -22.7 & & & \\
\hline HD 143347 & RS CrB & SRa & 332 & -81.5 & $0.5(2)$ & -85.5 & & & \\
\hline HD 150077 & TX Dra & $\mathrm{SRb}$ & 78 & 46.0 & $0.2(2)$ & 46.4 & & & \\
\hline HD 156163 & UW Her & $\mathrm{SRb}$ & 104 & -32.1 & & -29.3 & & 1.1 & \\
\hline HD 156205 & TT Dra & $\mathrm{SRb}$ & 107 & -15.4 & & -18.1 & & & \\
\hline HD 163990 & OP Her & $\mathrm{SRb}$ & 121 & 9.4 & & 7.4 & & & \\
\hline HD 166253 & V566 Her & $\mathrm{SRb}$ & 137 & -25.1 & & -23.3 & & & \\
\hline HD 168198 & IQ Her & $\mathrm{SRb}$ & 48 & -96.5 & $0.2(2)$ & -93.9 & & 0.3 & + \\
\hline HD 169931 & V988 Oph & $\mathrm{SRb}$ & 129 & -14.8 & $0.2(2)$ & -17.2 & & 0.3 & + \\
\hline HD 175865 & R Lyr & $\mathrm{SRb}$ & 46 & -28.4 & $0.7(2)$ & -29.3 & & & \\
\hline HD 178770 & V398 Lyr & $\mathrm{Lb}$ & 113 & -18.3 & & -18.9 & & 0.3 & \\
\hline HD 184313 & V450 Aql & $\mathrm{SRb}$ & 54 & -51.1 & & -53.8 & & 0.4 & \\
\hline HD 186352 & V1351 Cyg & $\mathrm{Lb}$ & 44 & -29.8 & $0.2(2)$ & -31.0 & & 0.4 & \\
\hline HD 217542 & BC And & $\mathrm{Lb}$ & - & -15.9 & & -15.7 & & 0.2 & + \\
\hline HD 229550 & MZ Her & $\mathrm{SRb}$ & 114 & 11.7 & & 11.0 & & & \\
\hline HIP 74704 & Z Ser & SRa & 88 & -21.1 & & -19.5 & & & \\
\hline HIP 84027 & CX Her & $\mathrm{SRb}$ & 97 & -56.3 & $1.5(2)$ & -57.5 & & 0.6 & \\
\hline- & V585 Oph & $\mathrm{SRb}$ & 144 & -59.4 & & -58.8 & & & \\
\hline- & V858 Aql & $\mathrm{Lb}$ & - & -15.2 & & -15.3 & & & \\
\hline
\end{tabular}

periods in a large fraction of the variables of our sample. These cases are marked in the last column of Tables 1 and 2. We will come back to this point later.

One clearly defined maximum or minimum close to the time of the two observing runs was selected to calculate phases. It has to be noted that these phases can still be somewhat erroneous as illustrated for the star RY CrB in Fig. 1. The fit through the data is based on two periods of 87 and 981 days, respectively, which were indicated by Fourier analysis. The GCVS period of this star is 90 days, i.e. close to the smaller period derived. We therefore used a period of 90 days in the following analysis. However, Fig. 1 clearly shows the existence of a second, much longer period. The long periods found can have similar or even larger amplitudes than the corresponding short period. It is therefore not trivial to decide which is the relevant period for the phase calculation. Note that especially the long periods could not be determined very accurately due to the small time span of the photometric monitoring compared to the period length.

Figure 1 also demonstrates that the light curve fit is far from perfect. It has been shown (e.g. Kiss et al. 1999; Kerschbaum et al. 2001; Lebzelter \& Kiss 2001), that a single period is in most cases not sufficient to reproduce the observed light curves. Both effects, long period and irregularities or multiperiodicity, limit the possibility to derive accurate phases for the stars of our sample.

\section{Results}

\subsection{Velocity shift}

First we want to compare the velocities measured in this study and the COM velocity listed in Table 1 . All together we obtained 60 measurements that we could compare to a COM velocity. The star $\tau^{4}$ Ser shows an extremely large infrared to 


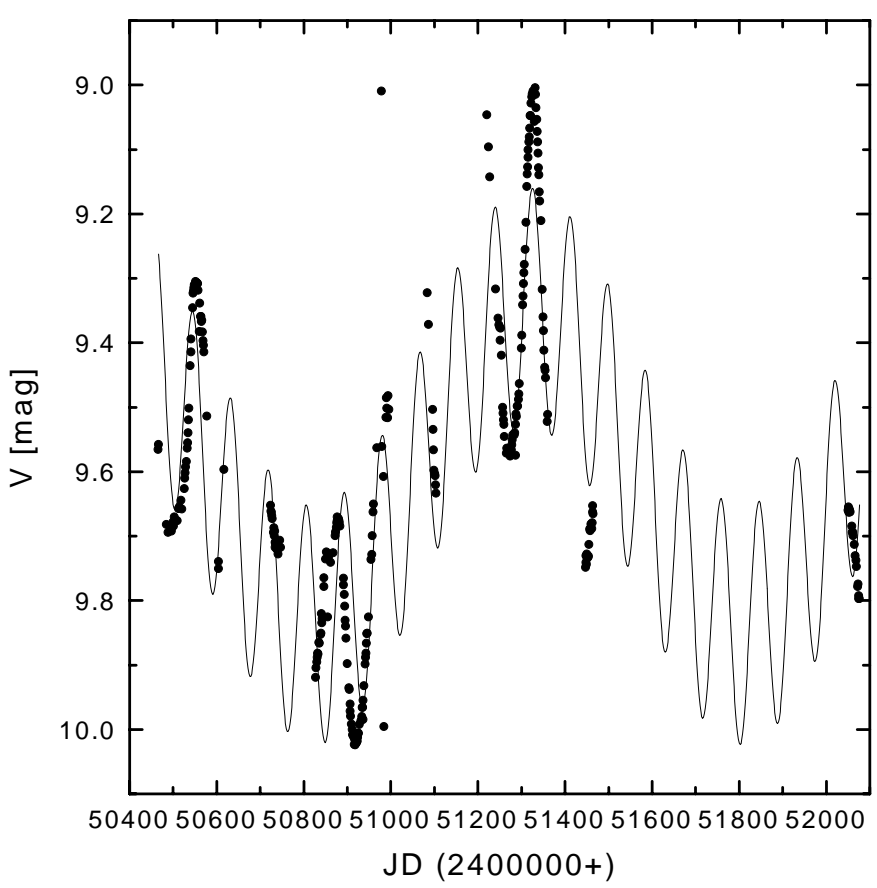

Fig. 1. Lightcurve data for the SRV RY CrB (dots) obtained with the APT. The solid line indicates a simple fit with two periods (87 and 981 days).

Table 3. Primary and secondary standards used. Column 4 gives the standard deviation of the star over one observing run. Velocity of the primary standards has been kept fixed during the data reduction, therefore no $\sigma$ is given.

\begin{tabular}{llrll}
\hline \hline HD number & name & $\begin{array}{c}\text { velocity } \\
{\left[\mathrm{km} \mathrm{s}^{-1}\right]}\end{array}$ & $\begin{array}{l}\sigma \\
\text { observing } \\
\text { run }\end{array}$ \\
\hline HD 81797 & $\alpha$ Hya & -4.45 & & April \\
HD 146051 & $\delta$ Oph & -19.64 & & April, July \\
HD 161096 & $\beta$ Oph & -12.30 & & April, July \\
\hline HD 81420 & 28 Hya & +4.4 & 0.2 & April \\
HD 82870 & 33 Hya & +13.2 & 0.2 & April \\
HD 113996 & 41 Com & +16.1 & 0.3 & April, July \\
HD 109317 & & -21.5 & 0.4 & April, July \\
HD 145897 & $\chi$ Sco & -23.4 & 0.1 & April \\
HD 148513 & & +7.8 & 0.3 & April, July \\
HD 169191 & & -19.8 & 0.2 & July \\
\hline
\end{tabular}

COM velocity difference of about $6 \mathrm{~km} \mathrm{~s}^{-1}$. Sato et al. (1978) and Yamashita \& Maehara (1979) note the occurrence of peculiar Balmer emission lines in the spectrum of $\tau^{4}$ Ser and suggest that this star is a close binary similar to $\mathrm{CH}$ Cyg. Binarity was also suggested by Young (1976) from radial velocity data. We therefore assume that the large velocity shift we observed is due to the orbital motion of the star within its system and exclude it from the further analysis. However, this star is definitely worth further investigation in order to reveal its nature.

From the remaining 24 stars which have been measured at two epochs, 16 objects or about $66 \%$ show in both measurements a velocity less than (i.e. blue shifted) or equal to the COM velocity. Among the stars measured only once 5 velocities are blue shifted and 5 are red shifted relative to the systemic (COM) velocity, in two cases the star's velocity matched

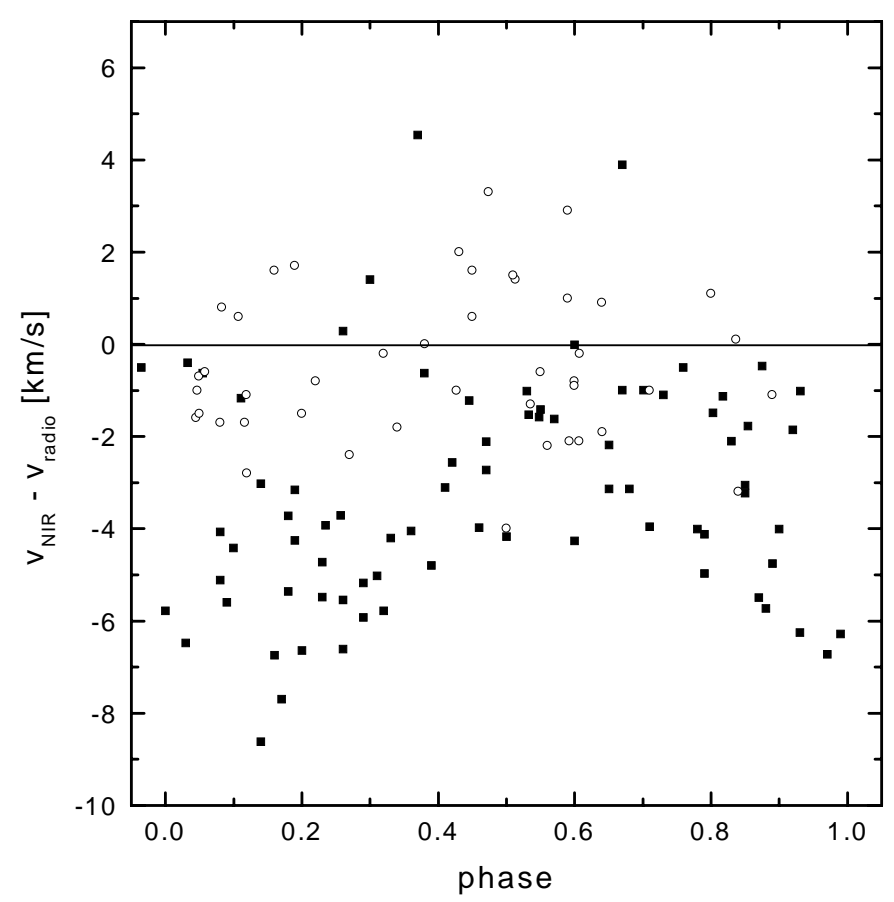

Fig. 2. Velocity shift of NIR velocity measurements relative to the systemic velocity $\left(v_{\mathrm{NIR}}-v_{\text {sys }}\right)$. Open circles denote new measurements from this paper, while black boxes mark data points from earlier papers. The straight line indicates the center-of-mass velocity.

the systemic velocity. No dependency of this result on the type of variability was found, but our sample included only 3 SRas, so that we could not derive any significant conclusions on that point.

In Fig. 2 we plot the difference between the measured velocity at $1.6 \mu \mathrm{m}$ and the COM velocity of the star. Phases have been determined as described above. Measurements from other papers (Hinkle et al. 1997; Lebzelter 1999) have been included with different symbols. Note that the data from these two previous papers represent only six different stars where velocity curves over one or several light cycles have been obtained.

Before we discuss this plot we want to recall the limitations in the accuracy of the data points in Fig. 2. Problems in deriving the correct phase have been noted above already. An estimation of the size of this error is difficult as it is impossible to predict the exact behavior of the light curve in these stars. To limit the error as much as possible we used only those stars in this plot where a maximum or minimum was measured within one light cycle of the spectroscopic observations. This reduced the number of usable measurements from 62 to 41 . As can be seen in Fig. 2 the remaining observations have been obtained at a wide variety of phases.

The uncertainty in the measured velocity is about $0.4 \mathrm{~km} \mathrm{~s}^{-1}$. No systematic shifts between observing nights have been found. We therefore assume that the main uncertainty in the velocity shift comes from the COM velocity. The uncertainty given by Kerschbaum \& Olofsson (1999) for COM velocities is about twice as large as the uncertainty of our measurements.

The plot reflects the findings already described. The majority of the measurements are blue shifted relative to the COM 
velocity. Two of the six previously discussed objects crossed the COM velocity. A similar fraction of the new data is found at positive velocity shifts (i.e. red shifted). Note that for one star from Hinkle et al. (1997), SV Peg, no good phase information was available and it was therefore not included in the plot. This star showed phases of both blue and red shifted near-infrared lines, so that the fraction of objects crossing the COM velocity in the older sample is slightly higher than in the present sample.

Figure 2 also reveals that the maximum difference between the systemic velocity and the near infrared CO lines in the present investigation is only about $4 \mathrm{~km} \mathrm{~s}^{-1}$, significantly smaller than the shifts found for some stars in the earlier papers. The blue shift in the new data is much less obvious than in the old ones. The median of the differences of measured and systemic velocity for the old data is about -3.1 , while the new data give only -0.8 .

Four of the seven stars previously observed were reobserved during the Phoenix observing runs in 2000. Two stars, RV Boo and g Her were observed in spring 1995 and 1996 (Lebzelter 1999) and always show blue shifted CO $1.6 \mu \mathrm{m}$ lines. During the observing runs in 2000, the velocities of $\mathrm{g} \mathrm{Her}$ were still smaller than the COM, while for RV Boo one spectrum was blue shifted and one red shifted. Between 1996 and 2000 Hinkle et al. (2002) measured the velocity of $g$ Her several times with a few measurements larger than the COM velocity. For the other two stars, SV Peg and W Cyg, the earlier measurements were obtained in 1984 to 1985 (Hinkle et al. 1997). The most interesting case is $\mathrm{W}$ Cyg, a star with puzzling velocities in the 1980 s that were shifted from the COM velocity by 3 to more than $6 \mathrm{~km} \mathrm{~s}^{-1}$. The velocities obtained in 2000 are much closer to the COM velocity. This supports the idea already suggested in Hinkle et al. (1997) that the velocities of this object are dominated by a long period variation.

\subsection{Velocity curve}

The data on the seven stars investigated previously suggested a typical shape of the velocity curve of SRVs (Lebzelter \& Hinkle 2002): the star reaches the most positive velocity around light minimum and the most negative velocity during maximum phase. This is in agreement with the comparison of light and velocity curves for individual stars presented by Lebzelter et al. (2000). While the new data roughly fit into this picture, an investigation of these data alone would not have suggested a general shape for the velocity curve this clearly. On the other hand for almost all stars where we have two observations the same tendency is seen, i.e. when the star changes from maximum towards minimum the velocity increases. This suggests that long secondary periods introduce an additional scatter in the velocity shifts plotted in Fig. 2 hiding the typical shape of the velocity curve of the semiregular and irregular variables (see below).

The maximum absolute velocity differences found for the stars of our sample scatter rather continuously between 0 and $4 \mathrm{~km} \mathrm{~s}^{-1}$ (excluding $\tau^{4}$ Ser). As pointed out above this shift includes not only the short period but also the long period variation. Ignoring the latter effect the typical amplitude of the velocity variation in SRVs would then be about 7 to $8 \mathrm{~km} \mathrm{~s}^{-1}$ (see Fig. 2). This about a factor of 2 larger than what was found for the majority of the SRVs that have been monitored over a whole light cycle (see Lebzelter 1999). We conclude that the total velocity amplitude is increased by the long period variations by about the same amount as the short period pulsations.

We studied the velocity variations further by using the extended sample (Table 2). The mean velocity difference for individual objects between April and July 2000 was $1.5 \mathrm{~km} \mathrm{~s}^{-1}$, i.e. clearly larger than the velocity error. The shift was on the average slightly larger for the extended sample compared to the core sample. This may be due to the on average longer periods of stars in the core sample, resulting in a smaller mean phase difference between the two observing runs. For the following discussion we will no longer separate the core sample from the extended sample.

Figure 3 shows the velocity difference between the two epochs of observation against the period. The two arrows mark a period of 87 and 174 days, respectively, which is one and two times the difference between the April and the July run. For stars with a period of 87 days the two measurements correspond to observations separated by one cycle, while for stars with a period of 174 days half a light cycle has passed between the two phases. Accordingly, one can see a trend of rising velocity difference in the period range from 87 to 174 days. Here the typical shape of the velocity curve of semiregular and irregular variables is much better defined than in Fig. 2 .

Five stars are separated from this trend. They are all found at periods between 150 and 200 days and at a small velocity difference. Two of these objects, AY Vir and RX Boo, may be explained by incorrectly reported or changed periods. For RT Vir and Y UMa our light curve data give some hint that during the observed light cycle the visual amplitude was lower than typical for these two stars. This may be reflected in a smaller velocity change.

A number of objects can be seen in the upper left corner of Fig. 3, i.e. stars with short periods and large velocity differences. This would suggest a similar velocity amplitude for stars in the period range from 50 to 200 days. We restricted Fig. 3 to periods below 250 days. A few objects of our sample could therefore not be plotted. The stars with periods greater than 250 days all show a velocity difference of more than $1 \mathrm{~km} \mathrm{~s}^{-1}$. $\operatorname{RSCrB}\left(P=332^{d}\right)$ has a velocity difference of $4 \mathrm{~km} \mathrm{~s}^{-1}$. The GCVS period has been confirmed by Kiss et al. (1999) who found the star to be monoperiodic. Variations with the same period have been seen in the velocity curve by Hinkle et al. (2002), who found a total velocity amplitude of about $6 \mathrm{~km} \mathrm{~s}^{-1}$. The time difference between the two observations corresponds to a phase difference of 0.26 . The results for this star are therefore in good agreement with earlier results. This may be a hint of a slight increase in the velocity amplitude towards longer periods.

\section{Conclusions}

The aim of this paper was to look for a possible blue shift of second overtone NIR CO lines, and to characterize the velocity 


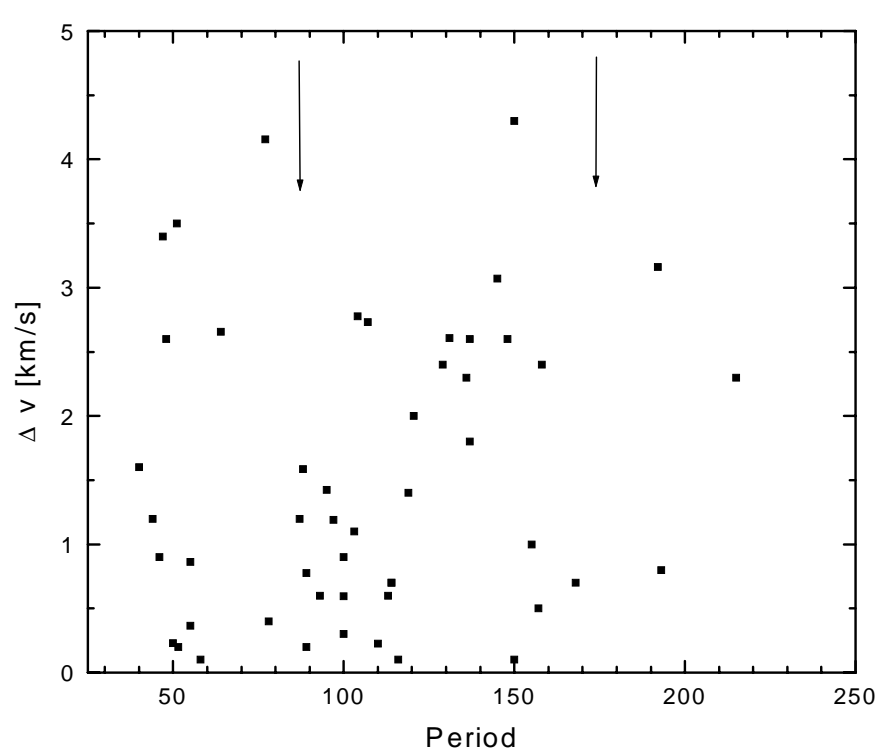

Fig. 3. Absolute velocity difference between April and July 2000 versus period for the core sample and the extended sample. The arrows indicate a period of 87 and 174 days, corresponding to the time between observations and twice this time.

variations in semiregular and irregular variables with the help of a large sample of stars. We summarize our results:

- We confirm an asymmetry of the NIR velocity curve relative to the center-of-mass velocity. Contrary to earlier investigations a significant number of SRVs and Lbs show velocities redshifted relative to the center-of-mass velocity.

- The typical SR velocity curve is continuous with an amplitude of approximately $3-4 \mathrm{~km} \mathrm{~s}^{-1}$. The velocity curve has a maximum around light minimum.

- No difference has been found in the behavior of the three subclasses of variables SRa, SRb and Lb.

- All SRVs show a similar spectrum in the $1.6 \mu \mathrm{m}$ range at all phases, which is clearly different from the very phase dependent mira spectra. An exception is the star V366 Aql which deserves further investigation (see appendix).

- Photometric evidence shows that multiple periodicity is very common among the SRVs. This complicates analysis of individual velocities or short time series of velocity measurements.

These results allow a number of conclusions: first, it can be excluded that large convective cells on the surface explain the light change of these stars in general. Lebzelter et al. (2000) have argued that if the movement of large convective cells is responsible for the variability of SRVs, one would never observe a velocity larger than the systemic velocity. As red shifted $\mathrm{CO}$ NIR lines have been observed in these stars we exclude this explanation. Other investigations (e.g. Lebzelter et al. 2000; Wood 2000) support the pulsation scenario, too. On the other hand, the role of long period variations is not clear yet (see below).

The shape and the asymmetry of the velocity curve is different from that of mira variables (e.g. Lebzelter \& Hinkle 2002). In miras (ignoring line doubling) the material is red shifted for the larger part of the light cycle. As discussed e.g. in
Lebzelter et al. (1999) this type of velocity curve can be reproduced by multiple shells at different distances from the pulsation driving zone. The shape of the velocity curve of SRVs is completely different from the discontinuous velocity curves found in miras. Despite the fact that emission lines have at some time been reported for several of the SRVs in our sample we did not find any indications of line doubling in these stars. Figure 2 also suggests rather a continuous than a discontinuous velocity curve for SRVs, although, of course, a different shape of the curve cannot be excluded for some individual objects (e.g. W Hya). A tempting, but as yet unprovable explanation for these differences is the mode of pulsation. Most SRVs are believed to be overtone pulsators while miras are fundamental mode pulsators (Wood 2000).

We can estimate the typical blue shift in SRVs and Lbs to about $1 \mathrm{~km} \mathrm{~s}^{-1}$ resulting from the mean value of the velocity shifts measured in this investigation. This is in agreement with the results from Lebzelter (1999). As we noted above, older data suggested a much larger blueshift. We think that the older data slightly overestimated the typical blue shift for the following reasons: The old dataset consists of measurements of seven objects. For one of them, SV Peg, most of the available velocity data are redshifted resulting in a redshifted velocity curve. Note that this star is not included in Fig. 2 as we do not have simultaneous light curve data. Two more stars of the old sample, g Her and RV Boo, show a blueshift of about $1.1 \mathrm{~km} \mathrm{~s}^{-1}$, i.e. very close to the mean value found from the new data. The blueshift for W Hya (Hinkle et al. 1997) is also probably overestimated due to insufficient phase coverage (see Fig. 9 in Lebzelter et al. 2000). For the remaining three stars of the old sample, W Cyg, $\mathrm{X}$ Mon and RU Cyg, phase coverage is sufficient to exclude that the blueshift of these objects at that time has been derived incorrectly. The example of W Cyg illustrates the strong influence of long period variations on the measured radial velocity. As noted above the velocity of this star obtained during the Phoenix observing runs in 2000 is much closer to the center of mass velocity than the old data. We therefore propose that the outstanding velocity shift in these stars is due to an extraordinary large amplitude long period velocity variation. However, it would be necessary to have a more complete phase coverage for a larger sample of stars to pin down the size of the blueshift more accurately.

The origin of the long period variation has been recently discussed by Wood (2000) and Hinkle et al. (2002). According to Wood two explanations are likely - orbital motion in a binary system or strange pulsation modes caused by an interaction of pulsation and convection. Long time velocity monitoring of a small sample of SRVs presented by Hinkle et al. (2002) favours pulsation. We estimate that the long period variations have a typical amplitude of the order of $4 \mathrm{~km} \mathrm{~s}^{-1}$. Taking into account the three extreme cases mentioned above, this amplitude may be even of the order of $6 \mathrm{~km} \mathrm{~s}^{-1}$, i.e. larger than the typical velocity amplitude of the short period variability. Long period variations are present in a large fraction of the semiregular and irregular variables.

Most of the semiregular and irregular variables are pulsating on a short period time scale of about 50 to 300 days. The velocity amplitude is about 3 to $4 \mathrm{~km} \mathrm{~s}^{-1}$. No clear dependency 
of the velocity amplitude with period could be detected. It is possible that this is the result of the strong irregularities in the light curves of these stars. As in the case of determining the period it would be necessary to monitor the velocity variations over a long time to find a possible correlation of velocity amplitude and period. Hinkle et al. (1997) have argued that a correlation between period and velocity amplitude may exist. Our results do not support correlation for SRVs with periods below about 250 days and velocity amplitudes below $5 \mathrm{~km} \mathrm{~s}^{-1}$ but we can not rule out such a correlation for SRVs with larger periods and larger velocity amplitudes. However, the number of SRVs with large velocity amplitudes is small.

Our investigation confirms the existence of a general blue shift of the average velocity. This blue shift can be attributed to either the short or the long period variations. The approach with observations at only two epochs does not allow a final decision on this question. However, the size of the blue shift found for SRVs and Lbs is similar to the convective blueshift in K giants (Allende Prieto et al. 2002). Understanding this blue shift is critical for the study of outflow dynamics and mass loss properties in these stars. As a first step this paper provides the typical value for this shift.

Acknowledgements. This work was supported by Austrian Science Fund Projects P14365-PHY, S7308-AST and S7301-AST. T.L. is also supported by the APART program of the Austrian Academy of Science. This research made use of the SIMBAD database operated by CDS in Strasbourg, France, NASA's Astrophysics Data System Bibliographic Services, and the STScI Digitized Sky Survey. We wish to thank the referee for several helpful comments.

\section{Appendix A: V366 Aql}

The spectrum of the star V366 Aql (SRa, $P=144.5^{d}$ ) clearly differs from those found for all other SRVs (Fig. A.1). The spectrum is rather typical for a mira-type variable. Cross correlation analysis indicates two separate velocity peaks. Inspection of the data reveals that the spectral features are broadened due to line doubling, which could actually be resolved for some lines. An example is marked in the figure. The velocity difference of the two components is about $14.5 \mathrm{~km} \mathrm{~s}^{-1}$. Note that the velocity split given is only an estimate since the spectral coverage is limited and the heavy line blending results in only a few measurable doubled lines. A similar result was found for both observing runs. The phase difference between the two observing runs is 0.6. Comparing this result and the similarity of the two spectra with typical velocity curves of miras (e.g. Lebzelter \& Hinkle 2002) we conclude that probably neither of the observations was taken during light maximum. The total velocity amplitude can therefore be expected to be greater than $14 \mathrm{~km} \mathrm{~s}^{-1}$. However, this is based on the assumption that V366 Aql is a misclassified mira-variable.

We therefore tried to check the classification of this star as SRV. The GCVS entry is based on the measurements by Hoffmeister et al. (1954). They describe the light curve as having variable shape with an amplitude of $1 \mathrm{mag}$. However, this is a lower limit set by the plate limit. The star is present on two POSS II-Red plates obtained in 1991 and 1992, respectively. The star's brightness changed between these two observations

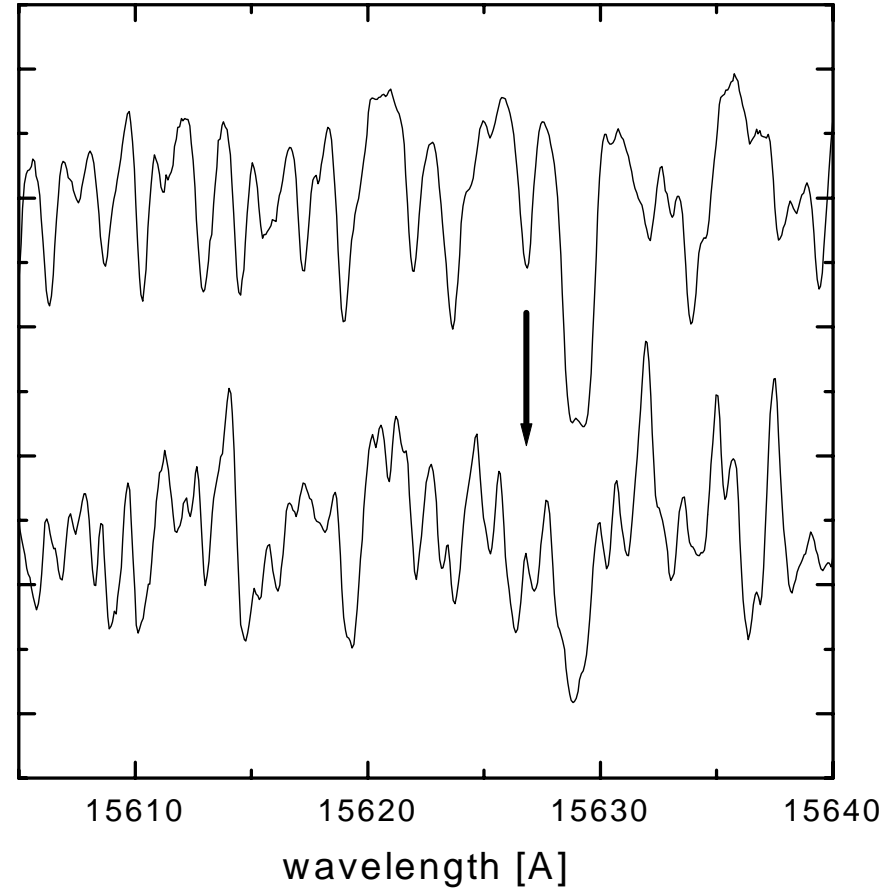

Fig. A.1. Spectrum of V366 Aql (bottom) compared with a typical SRV spectrum (S Dra, top). The arrow marks an example for line doubling (CO 3-0 R53).

by about 1.2 mag. Taking the period found by Hoffmeister et al. the phase difference is 0.3 . While the amplitude of the star can therefore assumed to be somewhat larger than the one given in the GCVS, the POSS II plates give no clear indication that V366 Aql is a mira. This depends highly on the shape of the light curve and the phase of the observation. We found no independent indications for the variability class of the star. Kerschbaum \& Hron (1992) obtained near infrared colours of this star and classified it as "red" SRV. Stephenson (1992) lists a spectral type of M7 for V366 Aql. This is an untypically late type for a short period mira. The late spectral type is in agreement with results from blackbody fits of photometry from the visual to the IRAS range presented by Hron et al. (1997). In this study V366 Aql is the star with lowest temperature in a sample of semiregular and mira variables.

The current results do not permit a decision on the variability type of V366 Aql. If it is a semiregular (or at least small amplitude) variable it would be the first one of this kind showing line doubling. If it is a mira, it would be the first "detection" of a mira by its velocity behavior, and it would be a very late spectral type case of a short period mira. In any case this star is clearly worthy of continued investigation.

\section{References}

Allende Prieto, C., Lambert, D. L., Tull, R. G., \& MacQueen, P. J. 2002, ApJ, 566, L93

Dravins, D. 1987, A\&A, 172, 200

Duflot, M., Figon, P., \& Meyssonnier, N. 1995, A\&AS, 114, 269

Edmonds, F. 1969, J. Quant. Spectrosc. Radiat. Transfer, 9, 1427

Gautschy, A. 1999, A\&A, 349, 209

Hinkle, K. H. 1978, ApJ, 220, 210 
Hinkle, K. H., \& Barnes, T. G. 1979, ApJ, 234, 548

Hinkle, K. H., Cuberly, R., Gaughan, N., et al. 1998, Proc. SPIE, 3354, 810

Hinkle, K. H., Hall, D. N. B., \& Ridgway, S. T. 1982, ApJ, 252, 697

Hinkle, K. H., Lebzelter, T., \& Scharlach, W. W. G. 1997, AJ, 114, 2686

Hinkle, K. H., Lebzelter, T., Joyce, R. R., \& Fekel, F. C. 2002, AJ, 123,1002

Hinkle, K. H., Wallace, L., \& Livingston, W. 1995, Infrared Atlas of the Arcturus Spectrum, 0.9-5.3 $\mu \mathrm{m}$ (San Francisco: Astronomical Society of the Pacific)

Hoffmeister, C., Ahnert-Rohlfs, E., Ahnert, P., et al. 1954, VSS, 2, 45

Hron, J., Kerschbaum, F., \& Aringer, B. 1997, A\&A, 322, 280

Kerschbaum, F., \& Hron, J. 1994, A\&AS, 106, 397

Kerschbaum, F., \& Olofsson, H. 1999, A\&AS, 138, 299

Kerschbaum, F., Lebzelter, T., \& Lázaro, C. 2001, A\&A, 375, 527

Kholopov, P. N., Samus, N. N., Frolov, M. S., et al. 1985-88, General Catalogue of Variable Stars, 4th edition, "Nauka" Publishing House, Moscow (GCVS)

Kiss, L. L., Szatmary, K., Cadmus, R. R., \& Mattei, J. A. 1999, A\&A, 346,542

Lebzelter, T. 1999, A\&A, 351, 644

Lebzelter, T., Hinkle, K. H., \& Aringer, B. 2001, A\&A, 377, 617

Lebzelter, T., \& Hinkle, K. H. 2002, in Proc. IAU Coll. 185, Radial and nonradial pulsations as probes of stellar physics, ed. C. Aerts, T. Bedding, \& J. Christensen-Dalsgaard, ASP Conf. Ser., 259, 556 Lebzelter, T., Hinkle, K. H., \& Hron, J. 1999, A\&A, 341, 224

Lebzelter, T., Kerschbaum, F., \& Hron, J. 1995, A\&A, 298, 159

Lebzelter, T., Kiss, L. L., \& Hinkle, K. H. 2000, A\&A, 361, 167

Lebzelter, T., \& Kiss, L. L. 2001, A\&A, 380, 388

Loidl, R., Höfner, S., Jørgensen, U. G., \& Aringer, B. 1999, A\&A, 342,531

de Medeiros, J. R., \& Mayor, M. 1999, A\&AS, 139, 433

Olofsson, H., González Delgado, D., Kerschbaum, F., \& Schöier, F. L. 2002, A\&A, in press

Sato, K., Kikuchi, I., Yamashita, Y., \& Norimiot, Y. 1978, PASJ, 30, 537

Skuljan, J., Hearnshaw, J. B., \& Cottrell, P. L. 2000, PASP, 112, 966

Sperl, M. 1998, Comm. Asteroseismology (Vienna), 111, 1

Stephenson, C. B. 1992, IBVS, 3800

Strassmeier, K. G., Boyd, L. J., Epand, D. H., \& Granzer, T. 1997, PASP, 109, 697

van Belle, G. T., Dyck, H. M., Benson, J. A., \& Lacasse, M. G. 1996, AJ, 112, 2147

Wallerstein, G., \& Dominy, J. F. 1988, ApJ, 326, 292

Wood, P. 2000, PASA, 17, 18

Yamashita, Y., \& Maehara, H. 1979, PASJ, 31, 307

Young, A. 1976, ApJ, 205, 182 